\title{
Evaluation of Antibiotic Sensitivity Pattern and Plasmid Isolation of Klebsiella Pneumoniae Isolates from Clinical Specimens in Sylhet City, Bangladesh
}

\author{
P. Bhattacharjee, M. Z. Alam*, S. M. A. Sayem \\ Department of Genetic Engineering and Biotechnology, Shah Jalal University of Science and \\ Technology, Sylhet-3114, Bangladesh
}

Received 2 April 2016, accepted in final revised form 29 September 2016

\begin{abstract}
Klebsiella pneumoniae is recognized as an emerging opportunistic bacterial pathogen of clinical relevance for its association with both community acquired and nosocomial infections. Moreover, increased resistance to common antimicrobial agents complicates and limits available therapeutic options. The aim of this study was to investigate an antibiotic sensitivity pattern of $K$. pneumoniae isolated from clinical samples against commonly used antibiotics and to focus on the associated therapeutic challenges. Presumptive $K$. pneumoniae isolates were collected from a variety of sources like urine sample, pus, wound swab etc. and further analyzed through standard microbiological methods. Following the identification of 23 bacterium isolates from the collected specimens, an antimicrobial sensitivity test was carried out. The results of this test indicated that the organisms possess diversity of susceptibility profiles towards the drugs tested. A high rate of resistance to the first line antibiotics like Amoxicillin (100\%), Vancomycin (90.90\%), Erythromycin $(83.33 \%)$, and Cotrimoxazole $(83.33 \%)$, designates a critical therapeutic challenge. However, inspite of a moderate sensitivity to the Gentamicin (43.5\%), increased number of intermediate sensitivity to the drugs tested clearly point towards the multi-drug resistance of the K. pneumoniae isolates. Plasmid profiling experiment revealed that the size of the isolated plasmids varied in length, ranging from 800-900bp.
\end{abstract}

Keywords: Klebsiella pneumoniae; Antibiotic sensitivity; Multi-drug resistance.

(C) 2017 JSR Publications. ISSN: 2070-0237 (Print); 2070-0245 (Online). All rights reserved. doi: http://dx.doi.org/10.3329/jsr.v9i1.27221 J. Sci. Res. 9 (1), 79-86 (2017)

\section{Introduction}

Emergence of antimicrobial resistance among enteric gram-negative bacteria is becoming a critical concern with rapid increase in multidrug resistant organisms. Multi resistance has not only complicated the antibacterial choice, in course of time they are posing vital challenges for medical microbiologists, infection control agencies and antimicrobial drug

*Corresponding author: mzalamgen@yahoo.com 
discovery scientists. Beta-lactam antibiotics are most commonly used to treat bacterial infections. Resistance of enterobacteriaceae to broad spectrum beta-lactam antibiotics via Extended Spectrum Beta Lactamase (ESBL) production is an increasing problem worldwide [1].

Typically, ESBL enzymes are plasmid mediated and have the unique property to hydrolyze oxyiminobeta-lactam agents like third generation cephalosporin [2] including the sulbectam and clavulenic acid combinations and monobactams such as aztreonam [3]. Therefore, gram negative bacteria harboring beta lactamase producing plasmids are significantly resistant to multiple drugs. Moreover, these plasmids, encoding resistance genes act as a scaffold and being mobile genetic elements allows easy transfer of themselves between different bacterial species and from one strain to other in vivo [4].

$K$. pneumoniae has emerged as one of the leading causes of hospital acquired infections, particularly amongst the patients of neonatal intensive-care unit causing severe mortality at a rate of $70 \%$ [5]. The occurrence of infections due to multidrug resistant strains has noticeably increased over the past few decades. According to the statistics of the centers for disease control and prevention, K. pneumoniae spp. accounts for $8 \%$ of endemic hospital infections and 3\% of epidemic outbreaks [6].

The increasing incidence of Klebsiella Pneumoniae Carbapenemases (KPCs) is a significant public health challenge. Recent analysis on the infection characteristics of the K. pneumoniae carbapenemase (KPC) showed that, the majority of infections were due to K. pneumoniae (89\%) [7]. The most common site of infection was blood (52\%) followed by pulmonary (32\%) and urine (10\%) [8]. In Bangladesh, a comparative study was conducted on the malnourished and healthy children's gut microflora revealed that in malnourished children, the pathogenic Klebsiella was 174-fold higher than their healthy counterpart [9]. Another study in Bangladesh also reported that K. pneumoniae and Escherichia coli have shown resistance to commonly used amoxicillin, levofloxacin, ciprofloxacine, ampicillin and ceftriaxone and a high resistance of $95 \%$ to azythromycin [10].

This study was conducted with the aim to isolate and identify $K$. pneumoniae from various clinical specimens and to evaluate the antibiotic sensitivity profile and corresponding severity of multidrug resistance towards the locally used antimicrobial agents. Based on the results of plasmid analysis, we tried to estimate a relationship to the antibiotic sensitivity pattern.

\section{Materials and Methods}

\subsection{Collection of bacterial isolates}

At least 50 no. of the human clinical samples of Klebsiella isolates used in the present investigation which was obtained from various sources like tracheal swabs, sputum, wound swabs, pus and urine samples. The patients were of different ages and both sexes and informed verbally to use their samples for research purpose. After collection, all 
isolates were tagged, sub cultured and stored at $4{ }^{\circ} \mathrm{C}$ in the microbiology laboratory of GEB department of Shahjalal University of Science and Technology, Bangladesh.

\subsection{Identification and characterization of pathogenic bacteria}

A total of 50 received specimens were subjected to standard microbiological methods and 23 isolates of Klebsiella pneumoniae species were identified. In brief, the clinical isolates were examined morphologically with Gram staining and the motility test was performed according to the hanging drop method. Several biochemical tests like Indole test, catalase test, MR-VP test, etc. were performed according to the Bergey's manual of determinative bacteriology [11] in order to identify bacterial species.

\subsection{Antimicrobial sensitivity testing}

The antibiotic susceptibility pattern of the identified $K$. pneumoniae isolates was performed in vitro by using a modified disk diffusion test of Kirby-Bauer method on Muller-Hinton agar as described by the clinical laboratory standard Institute [12]. Following antibiotic disks were used for the test: Amoxicillin (AMX $30 \mu \mathrm{g} \mathrm{disk}{ }^{-1}$ ), Ciprofloxacin (CIP $5 \mu \mathrm{g} \mathrm{disk}^{-1}$ ), Cotrimoxazole (Cot $30 \mu \mathrm{g} \mathrm{disk}^{-1}$ ), Erythromycin(ER 15 $\mu \mathrm{g} \operatorname{disk}^{-1}$ ), Gentamycin (GN $10 \mu \mathrm{g} \operatorname{disk}^{-1}$ ), Azithromycin (AZ $15 \mu \mathrm{g} \mathrm{disk}{ }^{-1}$ ), Vancomycin (VA $30 \mu \mathrm{g} \mathrm{disk}^{-1}$ ), Chloramphenicol (Chl $10 \mu \mathrm{g} \mathrm{disk}^{-1}$ ), Ceftazidime (CAZ $30 \mu \mathrm{g} \mathrm{disk}^{-1}$ ). The procedure involved measuring the diameter of the zone of inhibition that results from diffusion of the agent into the medium surrounding the disk. The bacterial culture adjusted to desired turbidity ( $0.5 \mathrm{McFarland}$ standards) was inoculated into Muller Hinton agar plate by streaking the swab over the entire sterile agar surface. Following this, antibiotic disks were evenly placed on the surface of the agar plate by using sterile forceps. After 24 hours of incubation, the diameter of the zone of inhibition was measured to determine the sensitivity pattern of the K. pneumoniae isolates.

\subsection{Plasmid isolation}

Plasmid DNA was isolated and purified by the alkaline lyses method [18]. Briefly, bacterial isolates were inoculated in a freshly prepared LB medium and incubated overnight at $37^{\circ} \mathrm{C}$ for overnight at $120 \mathrm{rpm}$ in shaker incubator. About $1 \mathrm{~mL}$ of the culture was taken into the Eppendorf tube and centrifuged at $15000 \mathrm{rpm}$ for $1 \mathrm{~min}$. Alkaline lyses solution 1, 2 and 3 were added and then centrifuged the tubes at $15000 \mathrm{rpm}$ at $4{ }^{\circ} \mathrm{C}$ for 5 min. An equal volume of phenol to chloroform was added and mixed by vortexing and then centrifuged the emulsion at $15000 \mathrm{rpm}$ for $2 \mathrm{~min}$. at microfuge tube and following this the aqueous upper phase was transferred to a fresh tube. Nucleic acids were precipitated and were collected by centrifugation at $15000 \mathrm{rpm}$ for 5 minutes at $4^{\circ} \mathrm{C}$. The pellet was dried completely and the nucleic acids were dissolved in $50 \mu \mathrm{l}$ of TE ( $\mathrm{pH} 8.0$ ) 
containing $20 \mu \mathrm{g} / \mathrm{mL}$ DNase free RNase and vortexed for a few seconds and stored at $20^{\circ} \mathrm{C}$.

The plasmid DNA was subjected to electrophoresis using $0.7 \%$ agarose gel in TBE buffer for about 90 min. 1KB DNA ladders were included for the estimation of plasmid sizes. The gel was viewed on an Ultraviolet trans-illuminator (UVP, high performance trans-illuminator; USA).

\section{Results}

During the study period all the klebsiella sp. were processed for identification and sensitivity testing. The $K$. pneumoniae cultures showed positive results for citrate utilization and catalase reaction. On the other hand, they showed negative results for Indole production. According to Burgay's manual of determinative bacteriology, the negative result in the final test of $\mathrm{H}_{2} \mathrm{~S}$ production determines Enterobacteriaceae. Therefore, the negative result in our TSI (Triple Sugar Iron Agar) test (for $\mathrm{H}_{2} \mathrm{~S}$ production) indicates the presence of $K$. pneumonia. All the clinical isolates that were confirmed to be $K$. pneumoniae were tested for antimicrobial sensitivity. On the basis of their sensitivity to antibiotics, isolates were categorized into three groups i.e. susceptible $(\mathrm{S})$, resistant $(\mathrm{R})$ and intermediate (I) [12].

Table 1. Biochemical characteristics of presumptive Klebsiella pneumonia.

\begin{tabular}{lc}
\hline Biochemical characteristics of isolates & Results obtained \\
\hline Gram staining & - \\
Indole test & - \\
Methyl Red test & - \\
Citrate test & + \\
TSI test $\left(\mathrm{H}_{2} \mathrm{~S}\right)$ & - \\
Catalase test & + \\
Oxidase test & - \\
Voges'-Pastkauer test & + \\
\hline
\end{tabular}

It was observed that the isolates of $K$. pneumoniae collected from different pathological specimen exhibits varying degree of sensitivity to different antimicrobials. Significant numbers of the isolates were found multidrug resistant. For the convenience of our study we examined the first five samples with Amoxicillin, Ciprofloxacin, Vancomycin, Ceftazidime, Gentamycin and found almost all of them to be resistant to Amoxicillin and Vancomycin, although some were susceptible to Ciprofloxacin. A notable number of isolates showed resistance to Vancomycin $90.91 \%$, Cotrimoxazole and Erythromycin $83.33 \%$. However, highest susceptibility was observed towards Gentamycin (47.8\%) and Ciprofloxacin (30.43\%). Intermediate sensitivity pattern was observed in case of Azithromycin and Chloramphenicol at $33.33 \%$ and $22.22 \%$ respectively while for Erythromycin at $15.67 \%$ (Fig. 1). 


\subsection{Plasmid analysis}

Plasmid analysis of $K$. pneumoniae isolates revealed that plasmid bands occurred randomly in these isolates and none of these plasmids was found to be common in all the isolates. The sizes ranged from $800 \mathrm{bp}$ to $900 \mathrm{bp}$ detected in most of the plasmid-harbored K. pneumoniae isolates.

Table 2. Antibiogram of isolated K. pneumonia.

\begin{tabular}{lllllll}
\hline \multirow{2}{*}{$\begin{array}{l}\text { Name of } \\
\text { antibiotics }\end{array}$} & \multirow{2}{*}{$\begin{array}{l}\text { Disc } \\
\text { concentration }\end{array}$} & $\begin{array}{l}\text { No. of } \\
\text { isolates }\end{array}$ & $\begin{array}{l}\text { Sensitivity } \\
\text { pneumoniae }\end{array}$ & pattern & of & isolated $K$. \\
\cline { 4 - 6 } & & 5 & 100 & - & $\% \mathrm{I}$ & $\% \mathrm{~S}$ \\
\hline Amoxicillin & $30 \mu \mathrm{g}$ & 23 & 65.2 & 8.7 & - \\
Ciprofloxacin & $5 \mu \mathrm{g}$ & 23 & 43.48 & 8.7 & 47.43 \\
Gentamycin & $10 \mu \mathrm{g}$ & 11 & 90.91 & - & 9.1 \\
Vancomycin & $30 \mu \mathrm{g}$ & 5 & 60 & 20 & 20 \\
Ceftazidime & $30 \mu \mathrm{g}$ & 18 & 50 & 33.33 & 16.67 \\
Azithromycin & $15 \mu \mathrm{g}$ & 18 & 83.33 & 11.11 & 5.55 \\
Cotrimoxazole & $30 \mu \mathrm{g}$ & 18 & 66.66 & 22.22 & 11.11 \\
Chloramphenicol & $30 \mu \mathrm{g}$ & 18 & 83.33 & 16.67 & - \\
Erythromycin & $15 \mu \mathrm{g}$ & 18 & &
\end{tabular}

Legend: \% R - Resistant percent, \% I - Intermediate percent, \% S - Susceptible percent.

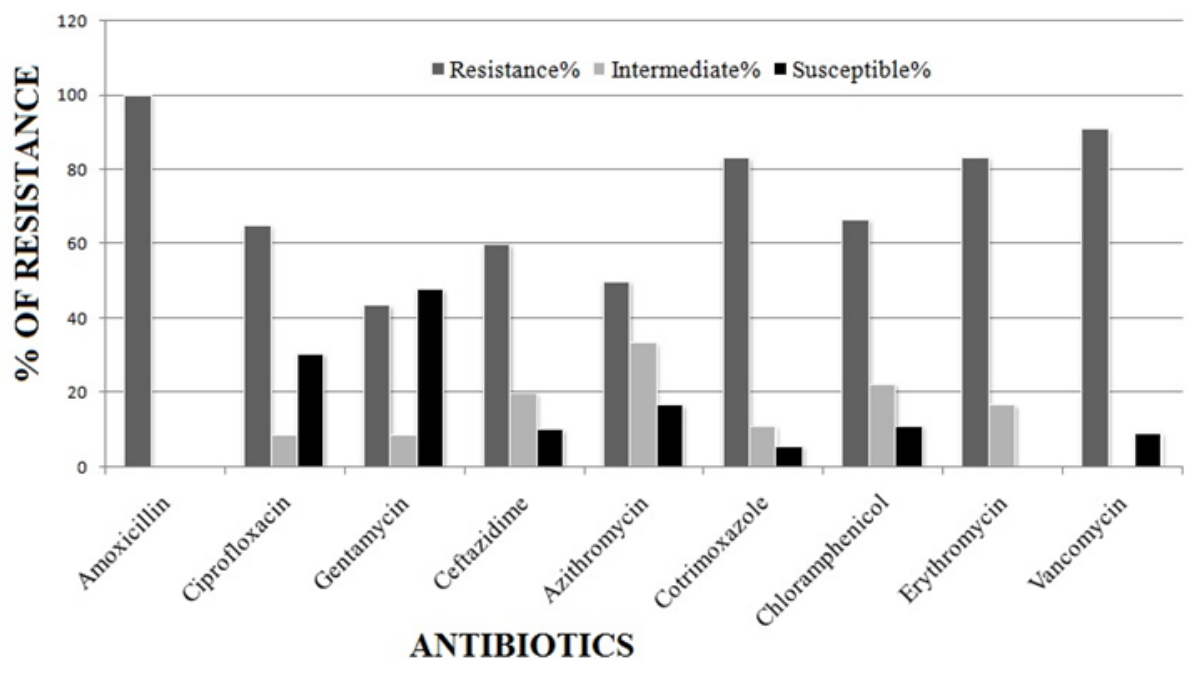

Fig. 1. Antibiotic resistance pattern of K. pneumoniae isolates.

\section{Discussion}

Resistance to antimicrobials is highly prevalent in bacterial isolates worldwide, particularly in developing countries. K. pneumoniae is also reported as a significant 
pathogen in nurseries, hospitals, communities and intensive care units despite many valuable antibiotics in use, and thus offering a challenge for the medical microbiologists to eradicate this problem.

The result of our present study indicated that most of the Klebsiella isolates possesses considerable resistance to the first line antibiotics such as Amoxicillin, Vancomycin, Cotrimoxazole, Erythromycin that are commonly prescribed by the physicians. The percent resistance of Amoxicillin (100\%) and Vancomycin (90.90\%) that observed were similar to the findings of another experiment conducted in our country which were $92 \%$ and $96 \%$ respectively [13]. In addition, amoxicillin, one of the frequently used medications for $K$. pneumoniae treatment, was found to be resistant at 88.5, 81.81 and $60.2 \%$ in the previous experiments $[14,15]$. The antibiotic resistance pattern observed is increasing at an alarming rate, posing a serious threat to the common therapeutic options.

Our investigation also revealed that the bacteria were not only resistant to Amoxicillin but also to Ciprofloxacin (65\%), a fluoroquinolone which is a commonly used for the treatment of bacterial infection. The resistance pattern of K. pneumoniae to Ciprofloxacin was reported to be $89 \%$ and $100 \%$ in two different studies [13]. Ciprofloxacin resistance pattern was lower in our experiment compared with these data. The percentage of antimicrobial resistance to Chloramphenicol $(66.67 \%)$ in this experiment corresponds with findings by Sikarwar, over $60 \%$ in India [16] but lesser than another study of Bangladesh where $76 \%$ resistance pattern was observed [13].

The antibiogram revealed that almost all isolates were resistant to Cotrimoxazole and Erythromycin in equal magnitude at $83.33 \%$. Similar research carried out in 2013, reported that about $52.8 \%$ of $K$. pneumoniae isolates showed resistance to Cotrimoxazole in India [15]. In 2012, data represented by Shahriar et al. from Bangladesh mentioned above $70 \%$ resistance to this drug. In comparison with both these results, our finding clearly illustrates a significantly higher resistance to this widely prescribed antibiotic. Another conspicuously highly resisted drug in our experiment is Ceftazidime at $70 \%$ of magnitude. This profile of resistance was remarkably higher than that recorded by Kumar [17] at $45.1 \%$ and $55.9 \%$ respectively. However, moderate level of susceptibility was reported for Gentamycin at $47.8 \%$.

These results clearly indicate that more attention is required to the ever increasing problem of drug resistance, since they are limiting the existing treatment options. Moreover, uses of broad spectrum antibiotics, insufficient aseptic condition and technique with inadequate control of infections spread had aggravated this problem. Not only $K$. pneumoniae, but some other strains of Klebsiella sp. are frequently encountered to possess resistance to the commercially available drugs and thus warrants due diligence in future surveillance efforts. Hence, preventative measures should be adopted to control the spread while ensuring faster identification system for $K$. pneumoniae with enhanced accuracy. Strict antibiotic policy should be adopted in hospitals to estimate the impact of higher resistance in bacteria and to take steps for reducing this resistance.

With a view to understanding the mechanism of antibiotic resistance, we focused at studying the changes in the molecular level. Plasmid isolation was performed and the 
bands were observed via agarose gel electrophoresis technique, where the plasmid bands ranges from 800-900 bp. In general, strains resistant to Ciprofloxacin contain plasmid DNA at $\pm 900 \mathrm{bp}$. Amoxicillin, that showed most resistance, have shown band at $\pm 900 \mathrm{bp}$ in most of the isolates. However, repeated experiment should need to confirm this statement.

\section{Conclusion}

The high level of resistance to the tested antibiotics dictates that such an emerging drug resistance pattern can complicate the therapeutic outcomes. In general, the relatively higher proportion of intermediate response of $K$. pneumoniae isolates to the antibiotics indicates the gradual migration to development of resistance of the microorganisms to these antibiotics. New strategies for antibiotic use and infection control, as well as further investigations on the mechanisms of antimicrobial resistance are crucial to the preservation of effective therapy against gram negative pathogens like K. pneumoniae.

\section{Acknowledgments}

This work was conducted and supported by the Microbiology laboratory, Department of Genetic Engineering and Biotechnology, Shahjalal University of science and technology, Bangladesh.

\section{References}

1. S. E. Mshana, E. Kamugisha, M. Mirambo, T. Chakraborty, and E. F. Lyamuya, BMC Res. Notes 49(2), (2009). http://dx.doi.org/10.1186/1756-0500-2-49

2. R. M. Parveen, M. A. Khan, G. A. Menezes, B. N. Harish, S. C. Parija, and J. P. Hays, The Ind. J. Med. Res. 134(3), 392 (2011).

3. S. Nathisuwan, D. S. Burgess, and J. S. Lewis, Pharmacotherapy 21(8), 920 (2001). http://dx.doi.org/10.1592/phco.21.11.920.34529

4. G. A. Jacoby and L. Sutton, Antimicrob. Agents Chemother. 35(1), 164 (1991). http://dx.doi.org/10.1128/AAC.35.1.164

5. M. E. Morgan, C. A. Hart, and R. W. Cooke, J. Hosp. Infect. 5, 377 (1984). http://dx.doi.org/10.1016/0195-6701(84)90005-7

6. U. Lundberg, B. M. Senn, W. Schüler, A. Meinke, and M. Hanner, Hum. Vac. Immun. 9(3), 497 (2013). http://dx.doi.org/10.4161/hv.23225

7. G. C. Lee and D. S. Burgess, Annals Clinical Microbiol. Antimicrob. 11, 32 (2012).

8. G. C. Lee, Pharm D, BCPS Epidemiology and Treatment of KPC'S... What's the news? Pharmacotherapy Rounds (2012).

9. S. Monira, S. Nakamura, K. Gotoh, K. Izutsu, H. Watanabe, N. H. Alam, and M. Alam, Front Microbiol. 228(2), (2011). http://dx.doi.org/10.3389/fmicb.2011.00228

10. M. A. Faiz and A. Basher, Antimicrobial Resistance: Bangladesh Experience, Regional Health Forum 15(1), 1 (2011).

11. J. G. Holt and D. H. Bergey, Bergey's Manual of Determinative Bacteriology, $9^{\text {th }}$ Edition, (Williams \& Wilkins Co, Baltimore, 1994). https://www.uiweb.uidaho.edu/micro_biology/250/IDFlowcharts.pdf 
12. CLSI Performance Standards for Antimicrobial Susceptibility Testing, Seventeenth Informational Supplement (M100-S17). Clinical and Laboratory Standards Institute, Wayne, PA. NCCLS 27(1), (2007). http://microbiolab- bg.com/wp-content/uploads/2015/05/CLSI.pdf

13. M. Shahriar, S. Mawla, S. M. A. Islam, and M. A. Bhuiyan, J. Sci. Res. 4(2), 499 (2012). http://dx.doi.org/10.3329/jsr.v4i2.8445

14. M. M. Feizabadi, G. Etemadi, M. Rahmati, S. M. Yeganeh, S. Shabanpoor, and S. Asadi, Nat Res Inst. Tuber Lung Dis. Iran Tanaffos 6(3), 20 (2007).

15. A. R. Kumar, Sch. J. App. Med. Sci. 1(6), 928 (2013).

16. A. S. Sikarwar and H. V. Batra - Int. Conf. Food Eng. Biotechnol. IPCBEE (Singapore, 2011) 9, pp. 130.

17. A. R. Kumar, J. Chem. Pharm. Res. 5(11), 326 (2013).

18. A. A. Olusola, I. J. Ogiogwa, O. O. Iheanyi, O. O. Phillip, C. N. Jonathan, and C. O. C. Charles, World App. Sci. J. 21(3), 371 (2013). 